\title{
Expression and Cellular Distribution of INHA and INHB before and after In Vitro Cultivation of Porcine Oocytes Isolated from Follicles of Different Size
}

\author{
Bartosz Kempisty, ${ }^{1,2}$ Marta Jackowska, ${ }^{3}$ Magdalena Woźna, ${ }^{3}$ Paweł Antosik, ${ }^{3}$ \\ Hanna Piotrowska, ${ }^{4}$ Piotr Zawierucha, ${ }^{1,2}$ Dorota Bukowska, ${ }^{3}$ Jędrzej M. Jaśkowski, ${ }^{3}$ \\ Michał Nowicki, ${ }^{1}$ and Klaus P. Brüssow ${ }^{5}$ \\ ${ }^{1}$ Department of Histology and Embryology, Poznan University of Medical Sciences, 6 Święcickiego St., 60-781 Poznań, Poland \\ ${ }^{2}$ Department of Anatomy, Poznań University of Medical Sciences, 6 Święcickiego St., 60-781 Poznań, Poland \\ ${ }^{3}$ Department of Veterinary, Poznań University of Life Sciences, 52 Wojska Polskiego St., 60-628 Poznań, Poland \\ ${ }^{4}$ Department of Toxicology, Poznań University of Medical Sciences, 30 Dojazd St., 60-631 Poznań, Poland \\ ${ }^{5}$ Department of Reproductive Biology, Leibniz Institute for Farm Animal Biology, Wilhelm-Stahl Allee 2, 18196 Dummerstorf, Germany \\ Correspondence should be addressed to Bartosz Kempisty, etok@op.pl
}

Received 24 July 2012; Revised 8 October 2012; Accepted 23 October 2012

Academic Editor: Leon Spicer

Copyright ( 2012 Bartosz Kempisty et al. This is an open access article distributed under the Creative Commons Attribution License, which permits unrestricted use, distribution, and reproduction in any medium, provided the original work is properly cited.

Cumulus-oocyte-complexes (COCs) were collected from small ( $<3 \mathrm{~mm})$, medium $(3-5 \mathrm{~mm})$, and large $(>5 \mathrm{~mm})$ porcine follicles, and the INHA and INHB expression and cellular localization were studied. Developmentally competent (BCB+) COCs were cultured for $44 \mathrm{~h}$. Samples of mRNA were isolated before and after in vitro maturation (IVM) from oocytes collected from follicles of different size for RQ-PCR assay. The INHA and INHB protein distribution within the oocytes was observed by confocal microscopy. INHA mRNA expression was increased in oocytes from large compared to medium and small follicles before IVM $(P<0.001)$, and to oocytes of small follicles after IVM $(P<0.001)$. The INHB expression was not different before IVM, but the IHNB mRNA level was gradually higher in oocytes from large follicles after IVM $(P<0.01)$. INHA was not differently expressed before IVM; however, in large follicle oocytes the protein was distributed in the peripheral area of the cytoplasm; in oocytes from small follicles it was in the entire cytoplasm. After IVM, INHA was strongly expressed in oocytes from small follicles and distributed particularly in the zona pellucida $(Z P)$. Similarly and both before and after IVM, INHB protein was highly expressed in small follicle oocytes and within the cytoplasm. In summary, INHs can be recognized as a marker of porcine oocyte quality.

\section{Introduction}

As yet, the oocyte developmental capacity in vitro, that is, to undergo maturation, fertilization, and to reach the blastocyst stage, has differed significantly from in vivo situation [1]. Though the in vitro cultivation (IVC) conditions try to mimic the in vivo environment, the maturation potential of the gametes is quite different. Although the efficiency of in vitro maturation (IVM) of porcine oocytes has been improved, there still exist problems with abnormal male pronucleus formation and an increased polyspermy rate [2]. It is accepted, that mammalian oocytes require both nuclear and cytoplasmic maturation to achieve developmental capacity [3-5]. Therefore, to optimize IVC systems in pigs, the determination of possible differences in a gene expression profile and/or cellular distribution of encoded proteins after IVC are of high interest.

Several intrinsic and extrinsic factors are associated with the maturation ability of porcine oocytes to reach MII stage $[5,6]$. In order to determine developmental competence of oocytes many authors use brilliant cresyl blue test (BCB) because of its simplicity and reliability relative to many species. The aim of the test is to evaluate activity of glucose-6-phosphate dehydrogenase (G6PDH) key enzyme 
of pentose phosphate pathway that produces ribose-5phosphate, erythrose-4-phosphate, and NADPH which are used in nucleotide synthesis, aromatic amino acid synthesis, and in reductive biosynthesis, respectively. Immature oocytes that require higher supplementation of energy to become developmentally competent will have higher concentration of enzyme in relation to matured oocytes. This will lead to reduction of $\mathrm{BCB}$ stain by $\mathrm{G} 6 \mathrm{PDH}$ in this cell resulting in colorless cytoplasm (cells described as $\mathrm{BCB}^{-}$). On the other hand, fully maturated oocytes have lower concentration of G6PDH that is insufficient to reduce the stain resulting in blue colorization of cytoplasm (cells described as $\mathrm{BCB}^{+}$).

Moreover, it was found that the follicular size is one of the factors which are linked with the expression of genes encoding proteins responsible for maturation and fertilization of oocytes. It was shown by Sun et al. (2001) [7] that the follicular size exerts no effect on the resumption of meiosis of oocytes recovered from small follicles, but it significantly affects the developmental potential of porcine oocytes. In addition, Antosik et al. [8] analyzed mRNAs expression of zona pellucida glycoproteins; pZP1, pZP2, pZP3, and pZP3 alpha; integrins ITGB1 and ITGB2 and pZP3 and ITGB2 proteins in porcine oocytes, isolated from follicles of various size. They found that the differential expression pattern of mRNAs and of encoded proteins, responsible for fertilization in pigs, was associated with the follicular size. Consequently, the ability of COCs which were recovered from distinct follicles may differ. Inhibins, described also as gonadal glycoprotein hormones, belong to the transforming growth factor beta (TGFB) superfamily and are involved in pituitary FSH secretion [9]. Inhibin exists in two forms, each comprising alpha subunit and covalently associated with one of two distinct subunits, respectively, termed inhibin beta-a (INHBA) and inhibin beta-b (INHBB). The genes encoding INHs are expressed in ovarian granulosa-cells. Their affected expression profile is an example of a primary marker of recurrent and residual ovarian granulosa-cell cancers [10]. Furthermore, mutation of INHA is the main cause of premature ovarian failure (POF) and other ovarian functional abnormalities [11-13]. In addition, Parrish et al. [14] compared expression of 20 different genes responsible for follicular growth in mouse ovarian follicles in vivo and cultured in vitro. They showed that expression of INHBA as well as expression of other members of TGF superfamily, including TGFB and BMP15, was downregulated in in vitro cultured follicles compared to in vivo.

However, only few reports are available which indicate expression of INHs in oocytes. Such an expression may suggest their role not only in folliculogenesis but also in oogenesis where TGFB superfamily proteins are involved in crucial steps of oocyte growth and achievement of developmental capacity $[15,16]$. Taking into account all of these data, the aim of this study was to analyze the expression of INHA and INHB mRNAs as well as the distribution of their encoded proteins in porcine oocytes recovered from follicles of different size before and after IVM.

\section{Material and Methods}

2.1. Animals. A total of 46 postpuberal crossbred Landrace gilts, with mean age of 155 days (range 140-170 days) and mean weight $100 \mathrm{~kg}(95-120 \mathrm{~kg})$ were used in this study. The experiments were approved by the local Ethics Committee.

2.2. Collection of Porcine Ovaries and COCs. The ovaries and reproductive tracts were recovered from gilts immediately after slaughter and transported to the laboratory within $20 \mathrm{~min}$ at $38^{\circ} \mathrm{C}$ in $0.9 \% \mathrm{NaCl}$. Follicles were classified into three size categories: small $(<3 \mathrm{~mm})$, medium $(3-5 \mathrm{~mm})$, and large $(>5 \mathrm{~mm})$ using a caliper.

The follicles were opened by individual puncturing with a $20-\mathrm{G}$ needle attached to a $5-\mathrm{mL}$ syringe into a sterile petri dish. The recovered cumulus-oocyte complexes (COCs) were washed three times in a modified PBS, supplemented with $36 \mu \mathrm{g} / \mathrm{mL}$ pyruvate, $50 \mu \mathrm{g} / \mathrm{mL}$ gentamycin, and $0.5 \mathrm{mg} / \mathrm{mL}$ bovine serum albumin (BSA; Sigma-Aldrich, St. Louis, MO, USA). They were selected under an inverted Zeiss microscope (Axiovert 35, Lübeck, Germany), counted, and morphologically evaluated with special care, using the scale suggested by Jackowska et al., (2009) [17]. Only COCs of grade I with a homogenous ooplasm and having uniform and compact cumulus cells were considered for use in the subsequent steps of the experiment.

2.3. Assessment of Oocyte Developmental Competence by Brilliant Cresyl Blue (BCB) Test. Before cultivation, COCs were washed twice in a modified Dulbecco PBS (DPBSm, Sigma-Aldrich), supplemented with $50 \mathrm{IU} / \mathrm{mL}$ penicillin, $50 \mu \mathrm{g} / \mathrm{mL}$ streptomycin (Sigma-Aldrich), 0.4\% [w/v] BSA, $0.34 \mathrm{mM}$ pyruvate, and $5.5 \mathrm{mM}$ glucose (DPBSm). Then, COCs were treated with $26 \mu \mathrm{M}$ brilliant cresyl blue (BCB; Sigma-Aldrich, St. Louis, MO, USA) diluted in DPBSm at $38.5^{\circ} \mathrm{C}$, under $5 \% \mathrm{CO}_{2}$ in air for $90 \mathrm{~min}$. After treatment, the oocytes were transferred to DPBSm and washed twice. During the washing procedure, the oocytes were examined under an inverted microscope and classified as either having stained blue $\left(\mathrm{BCB}^{+}\right)$or remaining colourless $\left(\mathrm{BCB}^{-}\right)$. Only $\mathrm{BCB}^{+}$oocytes, which had completed their growth phase and may have reached developmental competence, were used in the experiment.

2.4. In Vitro Maturation of Porcine COCs. The selected $\mathrm{BCB}^{+} \mathrm{COCs}$ were cultured in Nunclon $\Delta 4$-well dishes (Nunc, GmbH, Co. KG, Germany) in $500 \mu \mathrm{L}$ standard porcine in vitro maturation (IVM) medium (TCM-199 with Earle's salts, and L-glutamine, Gibco BRL Life Technologies, Grand Island, NY, USA) supplemented with $2.2 \mathrm{mg} / \mathrm{mL}$ sodium bicarbonate (Nacalai Tesque, Inc., Kyoto, Japan), $0.1 \mathrm{mg} / \mathrm{mL}$ sodium pyruvate (Sigma-Aldrich, St. Louis, MO, USA), $10 \mathrm{mg} / \mathrm{mL}$ BSA (Sigma-Aldrich), $0.1 \mathrm{mg} / \mathrm{mL}$ cysteine (Sigma-Aldrich), 10\% (v/v) filtered porcine follicular fluid, and gonadotropin supplements at final concentrations of $2.5 \mathrm{IU} / \mathrm{mL}$ human chorionic gonadotropin (hCG; Ayerst Laboratories, Inc., Philadelphia, PA, USA) and $2.5 \mathrm{IU} / \mathrm{mL}$ 
TABLE 1: Oligonucleotide sequences and primer probes used for RQ-PCR analysis.

\begin{tabular}{lcccc}
\hline Transcript & Sequence $\left(5^{\prime}-3^{\prime}\right.$ direction) & Probes number & Gene accession no. & Product size (bp) \\
\hline \multirow{2}{*}{ GAPDH } & F: TCCCTCAAGATTGTCAGCAA & 28 & NM001003142 & $68 \mathrm{bp}$ \\
& R: TGGATGACTTTGGCTAGAGGA & & & NM001003349 \\
-actin & F: GATCACCATTGGCAACGAG & 12 & & $111 \mathrm{bp}$ \\
& R: CCCAGGAAAGAAGGTTGGA & & NM001164842 & $101 \mathrm{bp}$ \\
INH B & F: ATCATCCAGGGGTCCTGTG & 26 & NM214189.1 & $105 \mathrm{bp}$ \\
& R: GACTGGGTGGGTCAACAAAG & &
\end{tabular}

equine chorionic gonadotropin (eCG; Intervet, Whitby, ON, Canada). Wells were covered with a mineral oil overlay and cells were cultured for $44 \mathrm{~h}$ at $38^{\circ} \mathrm{C}$ under $5 \% \mathrm{CO}_{2}$ in air. The COCs were incubated with bovine testicular hyaluronidase (BTH; Sigma-Aldrich, St. Louis, MO, USA) for $2 \mathrm{~min}$ at $38.5^{\circ} \mathrm{C}$ to separate the cumulus cells. These cells were removed by vortexing the oocytes. The cumulus cellfree oocytes were used for further analysis.

2.5. Confocal Microscopic Observations. Oocytes were isolated from small, medium, and large follicles (each $n=$ 10 per slide). Oocytes were fixed with $2.5 \%$ paraformaldehyde in PBS and $0.2 \%$ Triton-X 100 for $30 \mathrm{~min}$ at room temperature (RT) and washed three times in PBS/PVP $(0.2 \%)$. In order to block nonspecific binding, the samples were incubated with $3 \%$ BSA in PBS plus $0.1 \%$ Tween 20 for $30 \mathrm{~min}$ at RT. Oocytes were incubated for 12 hours at $4^{\circ} \mathrm{C}$ with goat polyclonal anti-INHA antibody (Ab, sc22048) or rabbit polyclonal anti-INHB (Ab, sc-50288) both from Santa Cruz Biotechnology (Santa Cruz, CA, USA), diluted 1:500 in $\mathrm{PBS} / 1.5 \% \mathrm{BSA} / 0.1 \%$ Tween 20 . After several washes with $\mathrm{PBS} / 0.1 \%$ Tween 20 , samples with goat polyclonal anti-INHA Ab were incubated for 1 hour at RT with fluorescein isothiocyanate (FITC)-conjugated anti-goat $\operatorname{IgG} \mathrm{Ab}$, produced in rabbits, and diluted $1: 200$ in $\mathrm{PBS} / 0.1 \%$ Tween 20 . Samples with rabbit polyclonal anti-INHB $\mathrm{Ab}$ were incubated for 1 hour at RT with crystalline tetramethylrhodamine isothiocyanate (TRITC)conjugated anti-rabbit IgG $\mathrm{Ab}$, produced in goats, and diluted $1: 200$ in $\mathrm{PBS} / 0.1 \%$ Tween 20 . Following washing in $\mathrm{PBS} / 0.1 \%$ Tween 20 , the oocytes were mounted on glass slides in antifade drop and observed under confocal system LSN 510 in Carl ZEISS microscope, Axiovert $200 \mathrm{M}$. FITC was excited with $488 \mathrm{~nm}$ from an argon laser, and emissions were imaged through a $505-530 \mathrm{~nm}$ filter. TRITC was excited with $543 \mathrm{~nm}$ from an argon laser and the emissions were imaged through a $565-615 \mathrm{~nm}$ filter.

2.6. Gene Expression Analysis. Total RNA was isolated from $\mathrm{BCB}^{+}$oocytes collected from large $(n=50)$, medium $(n=50)$, small follicles $(n=50)$ each before and after IVM using an RNeasy mini column Qiagen GmbH (Hilden, Germany). RNA samples were treated with DNAse I in order to purify the samples from DNA contamination, and the quality was examined by the microchannel electrophoresis using an Agilent Bioanalyzer (Agilent Technologies Inc., Palo Alto, CA). The RNA samples were resuspended in $20 \mu \mathrm{L}$ of RNase-free water and reverse-transcribed into cDNA. Realtime quantitative PCR amplification reactions were carried out in an AB 7500 HT Real-Time PCR System (Applied Biosystems) using Universal PCR Master Mix (Roche, Mannheim, Germany). All reactions were performed in a $20 \mu \mathrm{L}$ volume with 6 pmol forward and reverse primers and 4 pmol of probe (Table 1). The INHA and INHB expression was studied using primer probes (cat. no. 04687604001, cat. no. 04687574001, resp., Roche, Mannheim, Germany) according to the manufacturer's instructions. All reactions were executed in triplicates. In case of the negative control cDNA was not added. All primers used in the experiment were designed in the way that primers cover ends of two neighbour exons. In that case there was no need of performing reverse transcription control.

The relative expression levels of INHA and INHB transcripts in each sample were standardized by the internal standard of glyceraldehyde-3-phosphate dehydrogenase (GAPDH). In order to ensure that granulosa cells did not contaminate the oocytes, we demonstrated the absence of cytochrome P450 aromatase (cat. no. 69-04688686001), Roche, Mannheim, Germany) transcript by RT and RQ-PCR.

The two housekeeping genes GAPDH and $\beta$-actin (cat. no. 119-04693531001 and cat. no. 44-04688040001, resp., Roche, Mannheim, Germany) were amplified as the reference for mRNA quantification.

In order to quantify specific genes expressed in the oocytes, the levels of the expression of specific oocyte mRNAs in each sample were calculated relative to those of GAPDH and $\beta$-actin using the method described. Expression of GAPDH and $\beta$-actin mRNAs was measured in cDNA samples from isolated oocytes. All reactions were applied in at least five replicas. Determination of gene expression was based on $2^{\Delta \Delta \mathrm{Ct}}$ method according to Roche technical guide.

2.7. Statistical Analysis. Two-way ANOVA followed by the Tukey posttest was used to compare the results of the realtime PCR reaction. The differences were considered to be significant at $P<0.05, P<0.01$, and $P<0.001$. The software program GraphPad Prism version 4.0 (GraphPadSoftware, San Diego, CA, USA) was used for statistical calculations. 


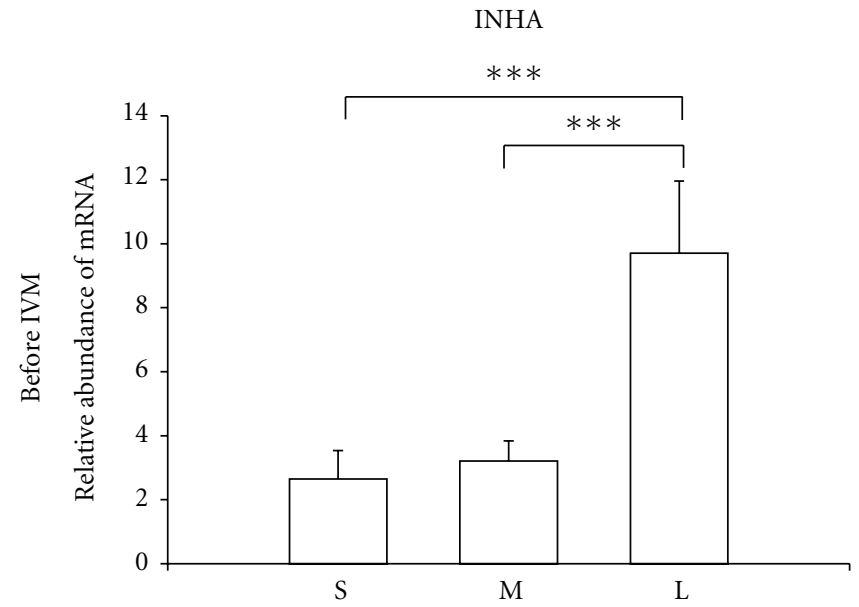

(a)

INHB

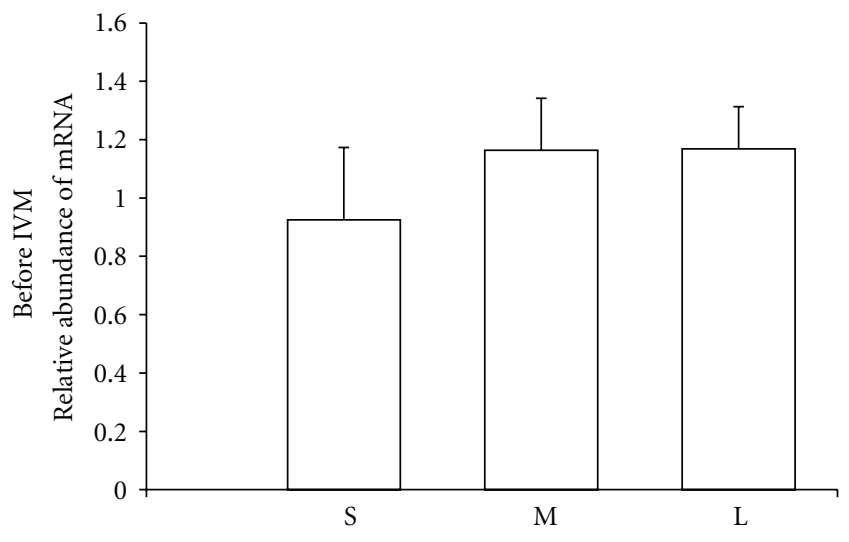

(c)

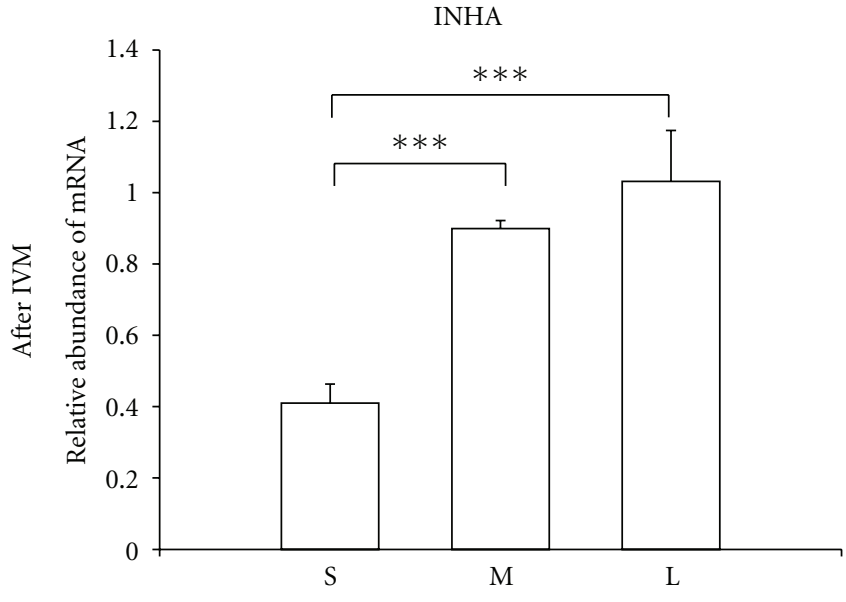

(b)

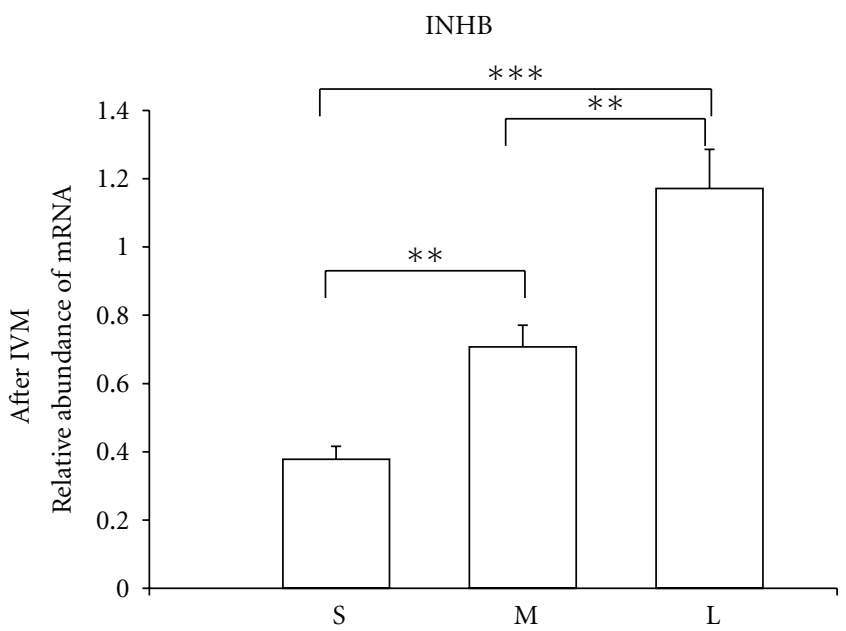

(d)

FIGURE 1: Relative abundance of INHA and INHB transcripts in porcine oocytes isolated from different size of follicles before and after IVM. The porcine oocytes were isolated from follicles of pubertal gilts and immediately used to isolate RNA, which was reverse-transcribed into cDNA. The relative abundance of INHA (a), (b) and INHB (c), (d) mRNAs was evaluated by RQ-PCR analysis before ((a), INHA, (c), INHB) and after ((b), INHA, (d), INHB) IVM. The oocytes analyzed before or after IVM were isolated from small $(<3 \mathrm{~mm})$, medium $(3-5 \mathrm{~mm})$ and large $(>5 \mathrm{~mm})$ follicles. Results are presented as mean \pm SEM with the level of significance, $* P<0.05,{ }^{* *} P<0.01,{ }^{* * *} P<0.001$.

\section{Results}

Several differences in the expression profile of INHA and INHB mRNAs as well as in localization and/or cellular distribution of encoded proteins were observed in oocytes collected from follicles of different size. Using RQ-PCR analysis we found an increased expression of INHA mRNA in oocytes isolated from large follicles as compared to oocytes from medium and small follicles before IVM $(P<0.001)$, (Figure $1(\mathrm{a}))$. When oocytes were analyzed after IVM, higher INHA mRNA level was revealed in oocytes isolated from large and medium follicles $(P<$ 0.001), (Figure 1(b)). To the contrary, no modifications were observed before IVM in the INHB mRNA expression profile between oocytes recovered from follicles of different size (Figures 1(c) and 1(d)). After IVM, a gradual increased expression of the mRNA was noted in oocytes with relation to the follicle size: large $>$ medium $>$ small follicles $(P<0.001, P<0.01, P<0.01$, resp.) (Figure $1(\mathrm{~d})$ ).

The confocal microscopy observations revealed no differences in INHA protein expression within the oocytes isolated from different sized follicles. However, in oocytes from large follicles before IVM, INHA protein was distributed in the peripheral area of the oocyte membrane, whereas in oocytes from medium and small follicles INHA protein was significantly stained in the cytoplasm (Figures 2(a), 2(b), and 2(c)). After IVM, INHA protein manifested a strong zona pellucida expression in oocytes from all follicles (Figures 2(d), 2(e), and 2(f)). Additionally, an increased expression was observed in zona pellucida of oocytes from small follicles (Figure 2(f)). Before IVM, INHB protein expression was higher in oocytes collected from small follicles (Figure 3(c)). INHB was distributed in the cytoplasm rather than in the zona pellucida of oocytes of all follicle 


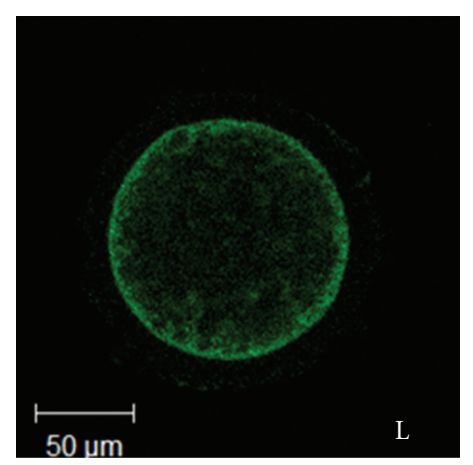

(a)

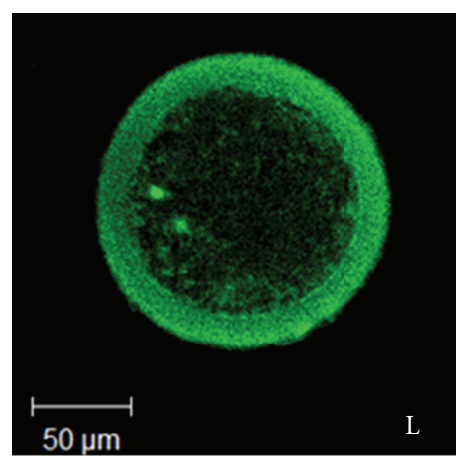

(d)

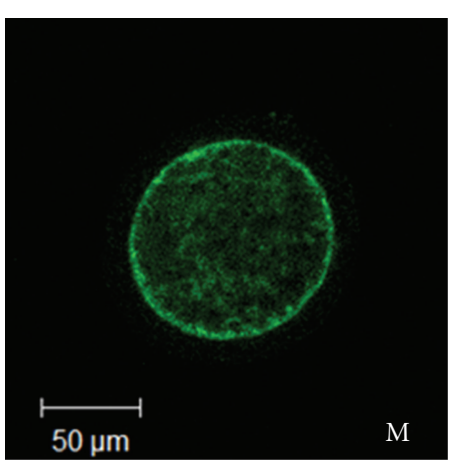

(b)

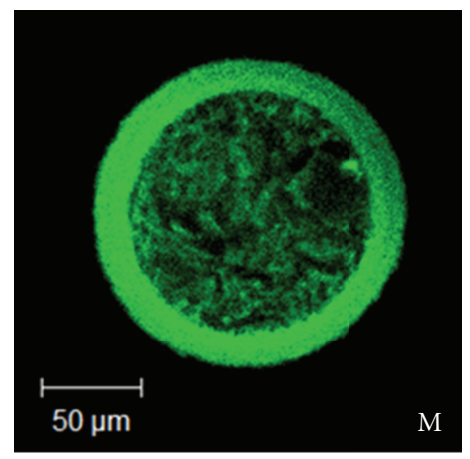

(e)

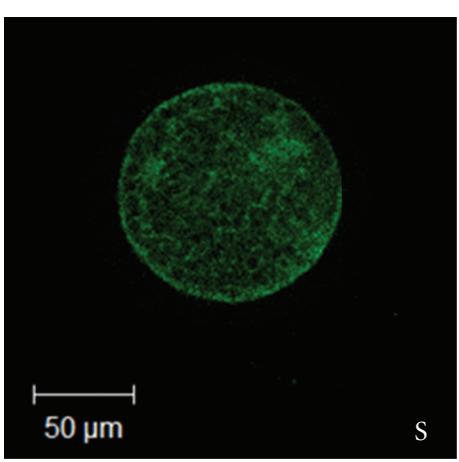

(c)

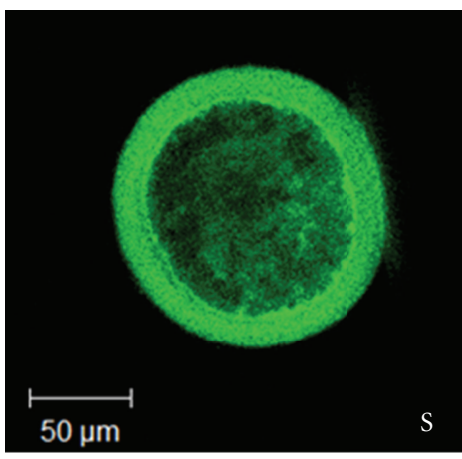

(f)

FIGURE 2: Confocal microscopic observation of INHA cellular distribution in porcine oocytes isolated from small, medium, and large follicles before and after IVM. Porcine oocytes isolated from small, medium, and large follicles were stained before and after IVM with porcine INHA (before IVM; (a), (b), and (c) and after IVM; (d), (e), and (f)), (goat polyclonal anti-INHA, sc-22048). The treated oocytes were labeled for 40 min with FITC-conjugated anti-goat IgG Ab, which emits a green fluorescent signal after excitation at $488 \mathrm{~nm}$ at a $1: 200$ dilution in PBS. Bars are $50 \mu \mathrm{m}$. L: large, M: medium, S: small follicles.

classes (Figures 3(a), 3(b), and 3(c)). There was an increase of INHB protein expression in oocytes from large follicles after IVM as compared to before IVM (Figure 3(d)). Overall, the highest expression of INHB was found in oocytes recovered from small follicles (Figure 3(f)). Furthermore, a distinct cytoplasmic distribution of INHB was noted in oocytes of all follicles groups (Figures 3(d), 3(e), and 3(f)). The negative control was performed without primary antibody against investigated proteins (Figures 4(a) and 4(b)).

\section{Discussion}

Porcine models are successfully applied in many fields of medicine including tissue engineering, cardiovascular disease, skin physiology, and reproductive biology (reviewed by [18]). Due to the good availability of porcine oocytes, they are of growing interest of studies at the molecular level of reproductive processes.

In several studies it was clearly shown that the maturation ability of mammalian oocytes is regulated on the molecular level by expression of genes and encoded by them proteins, and which are responsible for mitotic/meiotic progress and for morphological changes [19-22]. Genes belonging to TGFB superfamily are important regulators; they are called also "checkpoints of oocyte maturation potential" [23]. INHA and INHB are members of TGFB superfamily and it is suggested that they regulate important stages of oocyte maturation in vivo and in vitro [24-26]. However, the influence of several factors, for example, the follicular size and/or in vitro cultivation, on INHA and INHB mRNA expression profile or encoded proteins distribution within the porcine oocytes, is still not entirely known. Our study aimed to examine the role of the follicle size and IVC on the expression of INHA and INHB genes and the expression of respective proteins.

It has been shown in many species that the follicular size is correlated with developmental competence of oocytes. Despite differences in oocytes diameter across species used in the definition of small, medium, and large oocyte applied by authors, the general hypothesis is still consistent. Bovine oocytes are a good example of such a situation where differentiation in groups is not the same, but authors agree that follicles of a higher diameter contain more developmentally competent oocytes $[27,28]$. This trend is preserved in many other species including camel, dogs, and pigs [29, 30].

The follicular size has an influence on the expression profile of several genes involved in oocyte maturation and fertilization, too [8, 31, 32]. Glister et al. [33] investigated the role of genes of the TGFB superfamily, including INHA, 


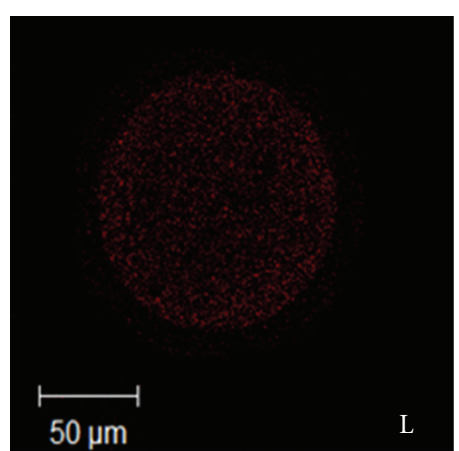

(a)

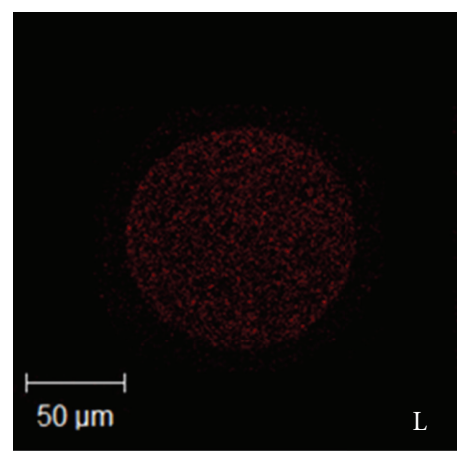

(d)

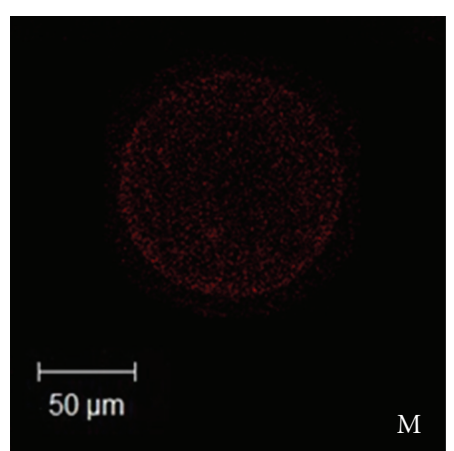

(b)

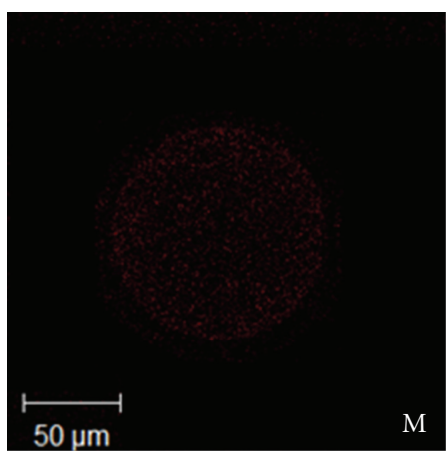

(e)

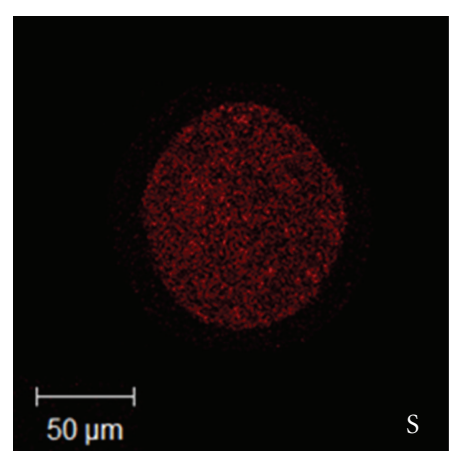

(c)

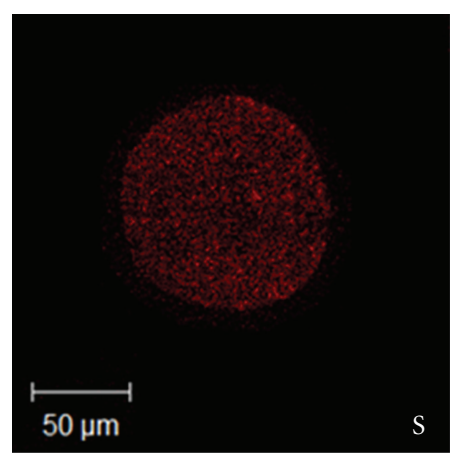

(f)

FIGURE 3: Confocal microscopic observation of INHB cellular distribution in porcine oocytes isolated from small, medium, and large follicles before and after IVM. Porcine oocytes isolated from small, medium, and large follicles were stained before and after IVM with porcine INHB (before IVM; (a), (b), and (c) and after IVM; (d), (e), and (f)), (rabbit polyclonal anti-INHB, sc-50288). The treated oocytes were labeled for $40 \mathrm{~min}$ with TRITC (crystalline tetramethylrhodamine isothiocyanate), which emits a red fluorescent signal after excitation at $543 \mathrm{~nm}$ at a $1: 200$ dilution in PBS for INHB. Bars are $50 \mu \mathrm{m}$. L: large, M: medium, S: small follicles.

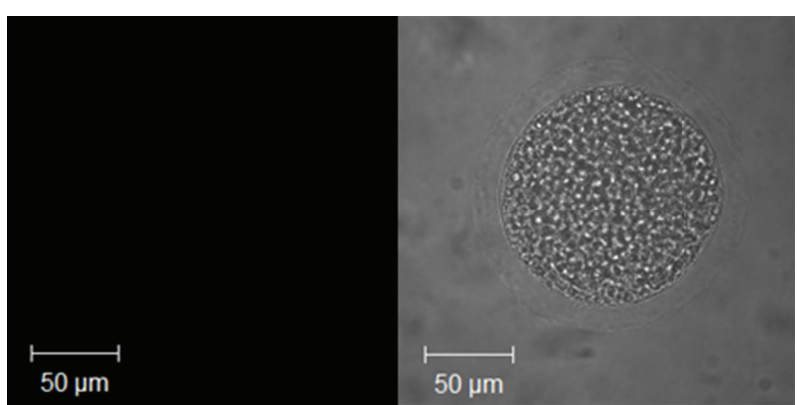

(a)

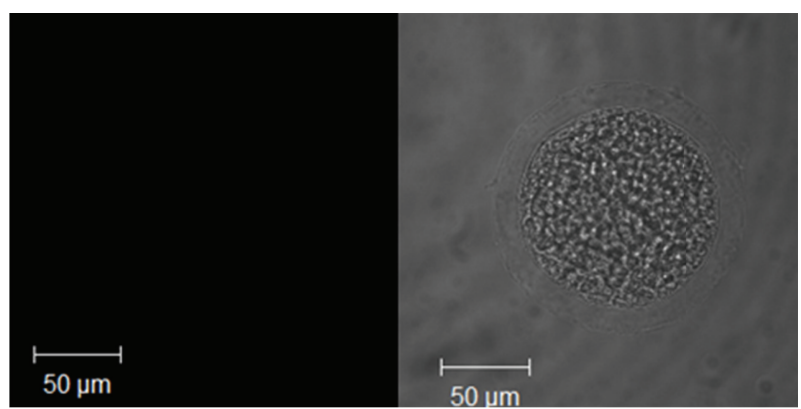

(b)

FIgURE 4: Confocal microscopic observation of control oocytes. Porcine oocytes with intact zona pellucida (negative control) were incubated for $1 \mathrm{~h}$ at room temperature with fluorescein isothiocyanate (FITC)-conjugated anti-goat IgG Ab produced in rabbits for INHA, diluted $1: 200$ in PBS/0.1\% Tween 20 (a), and for INHB with TRITC (crystalline tetramethylrhodamine isothiocyanate)-conjugated anti-rabbit IgG Ab produced in goats, at a 1:200 in PBS/0.1\% Tween 20 (b). The oocytes were mounted on glass slides in an antifade drop and observed under confocal system. Bars are $50 \mu \mathrm{m}$.

in the bovine follicular fluid in relation to the follicle growth. They found that each stage of the follicle growth was associated with the expression of INHA protein. Moreover, a stimulatory effect of FSH was noted on investigated genes. It may be suggested that the expression profile of INHA may represent a marker and prognostic factor of the follicle growth and development. These observations were partially proven by Jyotsna and Medhamurthy [34]. They analyzed changes in the estradiol and progesterone concentration in the Buffalo cow follicles during periovulatory development, and an increased expression of INHA in granulosa cells of the dominant follicle. Although there are only few published data regarding INHs expression and the growth of the follicle, the two experimental papers suggest that 
the growth of the follicle may be determined by INHA and upregulation of follicle development is associated with a higher expression profile of inhibins. Similarly, our study has revealed significant changes in both INHA and INHB mRNA expression profile in oocytes collected from porcine follicles of different sizes. Taking into consideration all these results, it may be suggested that INHs could be regarded as important factors involved in the porcine follicle growth too. Since it was documented in several studies that oocytes of an increased fertilization and maturation ability are recovered predominantly from large follicles $[8$, 31 ], our results have also shown that IHNs expression profile may be assumed to represent not only a marker of porcine follicle growth, but also a significant genetic index of the oocyte quality. Contrary to INHs mRNA expression profiles, we have observed an opposite effect of INHA and INHB protein expression and distribution in relation to the follicular size. An increased expression of these two proteins was detected in oocytes recovered from small follicles. This effect may be explained by the existence of different transcriptional and translational regulatory mechanisms and/or posttranscriptional modification of INHs mRNAs.

Although only one study describes the role of INHs in regulation of the cell cycle, the impact of other genes and encoded proteins in attainment of a full maturation by oocytes was recognized [35, 36]. Cyert and Kirschner [37] used Xenopus oocytes as an experimental model to study the activation of MPF and phosphorylation of pre-MPF and their involvement in the regulation of the cell cycle progress. They demonstrated that INH, as the pre-MPF inhibitor, has a function similar to protein phosphatase. Therefore, the activation of pre-MPF occurs via the mechanism of protein phosphorylation. The activation of INH is also regulated by another protein, called protein phosphatase-1. It is suggested that an upregulation of INH is necessary for MPF activation as a presumably first step of a mechanism that leads to the cell cycle regulation and progression. Since the cell cycle controlling system is a main mechanism associated with the maturation potential of mammalian oocytes, we investigated the INHA and INHB expression profiles in porcine oocytes before and after maturation in vitro. A substantially increased expression of INHA mRNA has been observed in oocytes before the maturation process; however, the INHB mRNA expression was unaffected. On the other hand, the expression of INHA and INHB proteins was significantly increased in oocytes after IVM. This observation points to the existence of a posttranscriptional regulation of INHs expression.

INHB protein was always distributed in the cytoplasm of porcine oocytes before and after IVM. Against, INHA protein was located in the peripheral area of the cytoplasm in oocytes before IVM, while after maturation the protein displayed a pronounced staining in the zona pellucida. An increased expression of INHA in the zona pellucida after IVM may indicate its role as an important gonadal glycoprotein. However, the crucial function of INHA at oocyte maturation and oocyte-sperm interaction needs to be confirmed in further experiments.

\section{Conclusions}

Our study demonstrated differential INHs mRNA expression profiles and distribution of the encoded proteins within porcine oocytes during the process of in vitro maturation. Furthermore, the relationship between the follicular size, oocyte maturation, and INHs expression profiling was shown. It is supposed that INHs are an important factor involved in the process of the follicle growth and oocyte maturation in pigs.

\section{Acknowledgment}

This study was made possible by Grants nos. NN 308588040 (2011/03/N/NZ4/00305) and 0233/IP1/2011/71 "IuventusPlus" from the Polish Ministry of Scientific Research and Higher Education.

\section{References}

[1] R. Moor and Y. Dai, "Maturation of pig oocytes in vivo and in vitro," Reproduction, vol. 58, pp. 91-104, 2001.

[2] T. Nagai, "The improvement of in vitro maturation systems for bovine and porcine oocytes," Theriogenology, vol. 55, no. 6, pp. 1291-1301, 2001.

[3] R. L. Krisher, "The effect of oocyte quality on development," Journal of Animal Science, vol. 82, no. 13, pp. 114-123, 2004.

[4] L. R. Martins, C. B. Ponchirolli, S. L. Beier, F. C. LandimAlvarenga, and M. D. Lopes, "Analysis of nuclear maturation in in vitro matured oocytes from estrous and anestrous bitches," Animal Reproduction, vol. 3, no. 1, pp. 49-54, 2006.

[5] Y. Yuan and R. L. Krisher, "in vitro maturation (IVM) of porcine oocytes," Methods in Molecular Biology, vol. 825, pp. 183-198, 2012.

[6] B. Kempisty, M. Jackowska, D. Bukowska et al., "Mechanisms regulating oogenesis, folliculogenesis and fertilization in pigs," Medycyna Weterynaryjna, vol. 67, no. 5, pp. 299-303, 2011.

[7] Q. Y. Sun, L. Lai, A. Bonk, R. S. Prather, and H. Schatten, "Cytoplasmic changes in relation to nuclear maturation and early embryo developmental potential of porcine oocytes: effects of gonadotropins, cumulus cells, follicular size, and protein synthesis inhibition," Molecular Reproduction and Development, vol. 59, no. 2, pp. 192-198, 2001.

[8] P. Antosik, B. Kempisty, D. Bukowska et al., "Follicular size is associated with the levels of transcripts and proteins of selected molecules responsible for the fertilization ability of oocytes of puberal gilts," Journal of Reproduction and Development, vol. 55 , no. 6, pp. 588-593, 2009.

[9] W. J. Bremner, "Inhibin: from hypothesis to clinical application," The New England Journal of Medicine, vol. 321, no. 12, pp. 826-827, 1989.

[10] S. Worbs, N. Shabani, D. Mayr et al., "Expression of the inhibin/activin subunits (-alpha, -betaA and -betaB) in normal and carcinogenic endometrial tissue: possible immunohistochemical differentiation markers," Oncology Reports, vol. 17, no. 1, pp. 97-104, 2007.

[11] N. Suzumori, S. A. Pangas, and A. Rajkovic, "Candidate genes for premature ovarian failure," Current Medicinal Chemistry, vol. 14 , no. 3, pp. 353-357, 2007.

[12] M. Myers, B. S. Middlebrook, M. M. Matzuk, and S. A. Pangas, "Loss of inhibin alpha uncouples oocyte-granulosa 
cell dynamics and disrupts postnatal folliculogenesis," Developmental Biology, vol. 334, no. 2, pp. 458-467, 2009.

[13] J. Hanrieder, A. Nyakas, T. Naessén, and J. Bergquist, "Proteomic analysis of human follicular fluid using an alternative bottom-up approach," Journal of Proteome Research, vol. 7, no. 1, pp. 443-449, 2008.

[14] E. M. Parrish, A. Siletz, M. Xu, T. K. Woodruff, and L. D. Shea, "Gene expression in mouse ovarian follicle development in vivo versus an ex vivo alginate culture system," Reproduction, vol. 142, no. 2, pp. 309-318, 2011.

[15] R. B. Gilchrist and L. J. Ritter, "Differences in the participation of TGFB superfamily signalling pathways mediating porcine and murine cumulus cell expansion," Reprduction, vol. 142, no. 5, pp. 647-657, 2011.

[16] R. A. Dragovic, L. J. Ritter, S. J. Schulz et al., "Oocyte-secreted factor activation of SMAD 2/3 signaling enables initiation of mouse cumulus cell expansion," Biology of Reproduction, vol. 76, no. 5, pp. 848-857, 2007.

[17] M. Jackowska, B. Kempisty, P. Antosik et al., "The morphology of porcine oocytes is associated with zona pellucida glycoprotein transcript contents," Reproductive Biology, vol. 9, no. 1, pp. 79-85, 2009.

[18] J. K. Lunney, "Advances in swine biomedical model genomics," International Journal of Biological Sciences, vol. 3, no. 3, pp. 179-184, 2007.

[19] T. Chu, I. Dufort, and M. A. Sirard, "Effect of ovarian stimulation on oocyte gene expression in cattle," Theriogenology, vol. 77, no. 9, pp. 1928-1938, 2012.

[20] Y. Yuan, M. B. Wheeler, and R. L. Krisher, "Disrupted redox homeostasis and aberrant redox gene expression in porcine oocytes contribute to decreased developmental competence," Biology of Reproduction, vol. 87, no. 4, p. 78, 2012.

[21] F. Cillo, T. Brevini, S. Antonini, A. Paffoni, G. Ragni, and F. Gandolfi, "Association between human oocyte developmental competence and expression levels of some cumulus genes," Reproduction, vol. 134, no. 5, pp. 645-650, 2007.

[22] H. Shao, C. Ma, X. Zhang et al., "Aurora B regulates spindle bipolarity in meiosis in vertebrate oocytes," Cell Cycle, vol. 11, no. 14, pp. 2672-2680, 2012.

[23] V. M. Jackowska, B. Kempisty, M. Woźna et al., "Differential expression of GDF9, TGFB1, TGFB2 and TGFB3 in porcine oocytes isolated from follicles of different size before and after culture in vitro," Acta Veterinaria Hungarica, vol. 61, no. 1, pp. 170-178, 2013.

[24] A. K. Nagaraja, B. S. Middlebrook, S. Rajanahally et al., "Defective gonadotropin-dependent ovarian folliculogenesis and granulosa cell gene expression in inhibin-deficient mice," Endocrinology, vol. 151, no. 10, pp. 4994-5006, 2010.

[25] N. Suzumori, S. A. Pangas, and A. Rajkovic, "Candidate genes for premature ovarian failure," Current Medicinal Chemistry, vol. 14, no. 3, pp. 353-357, 2007.

[26] C. L. Chang, T. H. Wang, S. G. Horng, H. M. Wu, H. S. Wang, and Y. K. Soong, "The concentration of inhibin B in follicular fluid: relation to oocyte maturation and embryo development," Human Reproduction, vol. 17, no. 7, pp. 17241728, 2002.

[27] P. Lonergan, P. Monaghan, D. Rizos, M. P. Boland, and I. Gordon, "Effect of follicle size on bovine oocyte quality and developmental competence following maturation, fertilization, and culture in vitro," Molecular Reproduction and Development, vol. 37, no. 1, pp. 48-53, 1994.

[28] P. Blondin and M. A. Sirard, "Oocyte and follicular morphology as determining characteristics for developmental competence in bovine oocytes," Molecular Reproduction and Development, vol. 41, no. 1, pp. 54-62, 1995.

[29] H. Khatir, A. Anouassi, and A. Tibary, "Effect of follicular size on in vitro developmental competence of oocytes and viability of embryos after transfer in the dromedary (Camelus dromedarius)," Animal Reproduction Science, vol. 99, no. 3-4, pp. 413-420, 2007.

[30] N. Songsasen and D. E. Wildt, "Size of the donor follicle, but not stage of reproductive cycle or seasonality, influences meiotic competency of selected domestic dog oocytes," Molecular Reproduction and Development, vol. 72, no. 1, pp. 113-119, 2005.

[31] M. Machatkova, K. Krausova, E. Jokesova, and M. Tomanek, "Developmental competence of bovine oocytes: effects of follicle size and the phase of follicular wave on in vitro embryo production," Theriogenology, vol. 61, no. 2-3, pp. 329-335, 2004.

[32] M. A. Bagg, M. B. Nottle, D. T. Armstrong, and C. G. Grupen, "Relationship between follicle size and oocyte developmental competence in prepubertal and adult pigs," Reproduction, Fertility and Development, vol. 19, no. 7, pp. 797-803, 2007.

[33] C. Glister, N. P. Groome, and P. G. Knight, "Bovine follicle development is associated with divergent changes in activin$\mathrm{A}$, inhibin-A and follistatin and the relative abundance of different follistatin isoforms in follicular fluid," Journal of Endocrinology, vol. 188, no. 2, pp. 215-225, 2006.

[34] U. R. Jyotsna and R. Medhamurthy, "Standardization and validation of an induced ovulation model system in buffalo cows: characterization of gene expression changes in the periovulatory follicle," Animal Reproduction Science, vol. 113, no. 1-4, pp. 71-81, 2009.

[35] B. Kempisty, M. Wozna, H. Piotrowska et al., "The expression of genes encoding zona pellucida glycoproteins in canine cumulus-oocyte complexes cultured in vitro in media supplemented with progesterone and estradiol," Theriogenology, vol. 77, no. 3, pp. 684-693, 2012.

[36] B. Kempisty, P. Antosik, D. Bukowska et al., "Assessment of zona pellucida glycoprotein and integrin transcript contents in porcine oocytes," Reproductive Biology, vol. 9, no. 1, pp. 7178, 2009.

[37] M. S. Cyert and M. W. Kirschner, "Regulation of MPF activity in vitro," Cell, vol. 53, no. 2, pp. 185-195, 1988. 

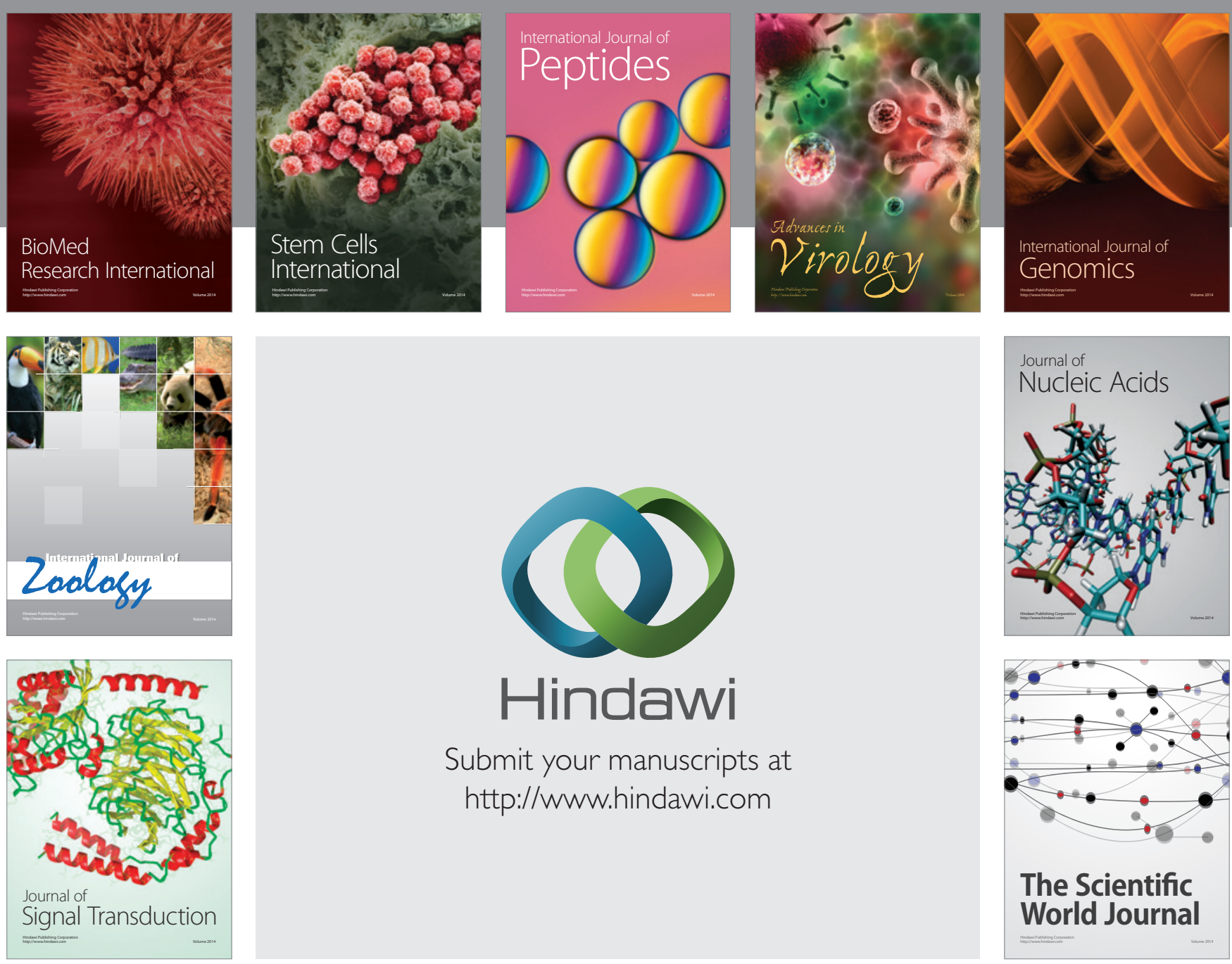

Submit your manuscripts at

http://www.hindawi.com
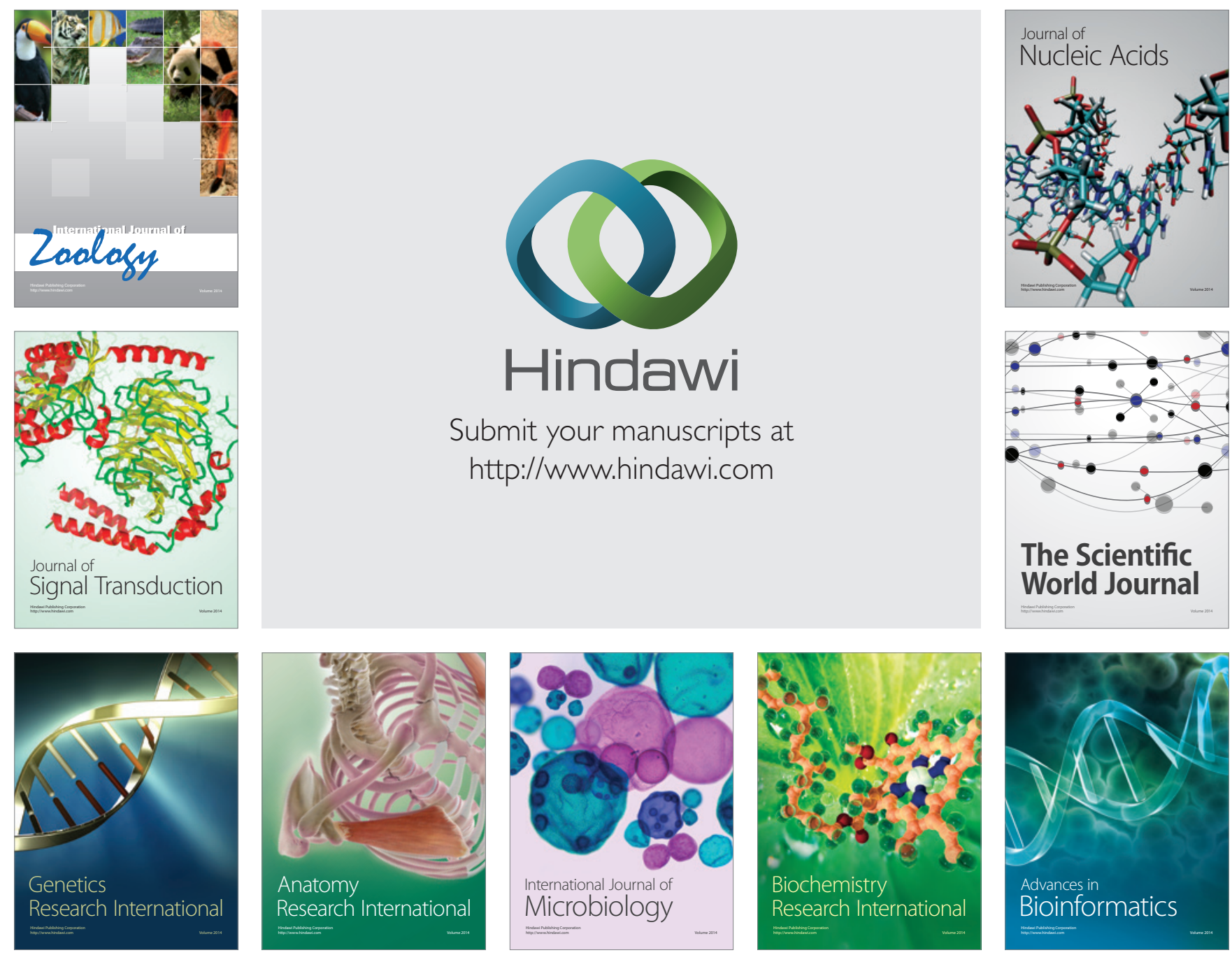

The Scientific World Journal
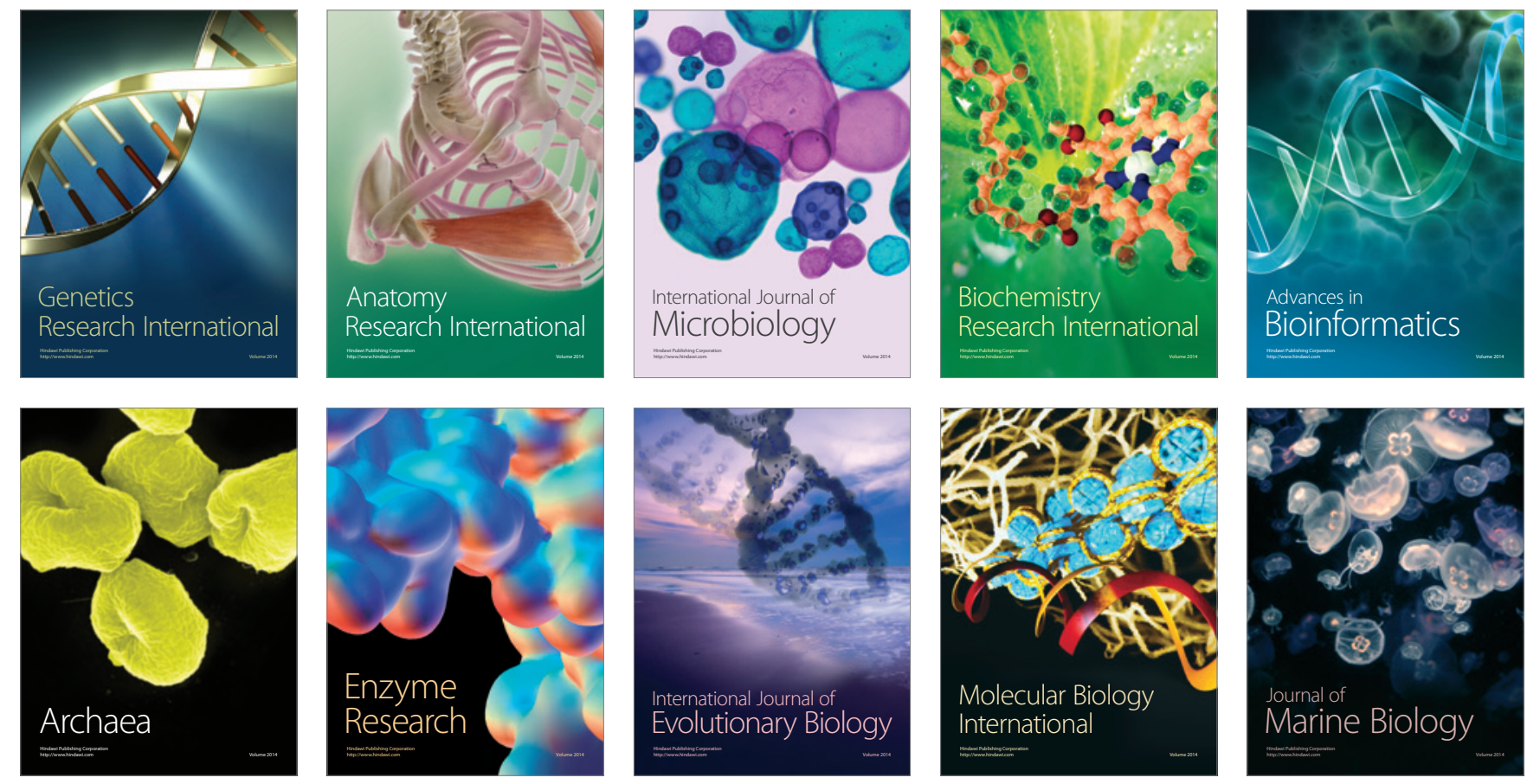